\title{
Outcomes of Combined Peritoneal and Local Treatment for Patients with Peritoneal and Limited Liver Metastases of Colorectal Origin: A Systematic Review and Meta-Analysis
}

\begin{abstract}
Margot C. E. Polderdijk, BSc ${ }^{1}$ (D), Max Brouwer, BSc ${ }^{1}$, Leonie Haverkamp, MD, PhD ${ }^{1}$, Kirsten A. Ziesemer, PhD $^{2}$, Mark Tenhagen, $\mathrm{MD}^{1}$, Djamila Boerma, $\mathrm{MD} \mathrm{PhD}^{3}$, Niels F. M. Kok, MD, PhD ${ }^{4}$, Kathelijn S. Versteeg, $\mathrm{MD}^{5}$, Dirkje W. Sommeijer, MD, PhD $^{5,6}$, Pieter J. Tanis, MD, PhD $^{1}$, and Jurriaan B. Tuynman, MD, PhD ${ }^{1}$

${ }^{1}$ Department of Surgery, Amsterdam University Medical Centers, Amsterdam, The Netherlands; ${ }^{2}$ Medical Library, Vrije Universiteit, Amsterdam, The Netherlands; ${ }^{3}$ Department of Surgery, St. Antonius Hospital, Nieuwegein, The Netherlands; ${ }^{4}$ Department of Surgery, Netherlands Cancer Hospital, Amsterdam, The Netherlands; ${ }^{5}$ Department of Medical Oncology, Amsterdam University Medical Centers, Amsterdam, The Netherlands; ${ }^{6}$ Department of Medical Oncology,
\end{abstract} Flevoziekenhuis, Almere, The Netherlands

\begin{abstract}
Background. Almost half of all colorectal cancer (CRC) patients will experience metastases at some point, and in the majority of cases, multiple organs will be involved. If the peritoneum is involved in addition to the liver, the current guideline-driven treatment options are limited. The reported overall survival ranges from 6 to 13 months for the current standard of care (systemic treatment). This study aimed to evaluate morbidity and clinical long-term outcomes from a combined local treatment of hepatic metastases with cytoreductive surgery (CRS) and hyperthermic intraperitoneal chemotherapy (HIPEC) used to treat peritoneal metastases.
\end{abstract}

Methods. A systematic search was performed in PubMed, Embase.com, Web of Science, and Cochrane. Studies evaluating the clinicopathologic data of patients who had both peritoneal and hepatic metastases treated with CRSHIPEC were included provided sufficient data on the primary outcomes (overall and disease-free survival) were presented. The quality of included studies was assessed using the Methodological Index for Non-Randomized Studies (MINORS).

(c) The Author(s) 2021

First Received: 19 April 2021

Accepted: 24 September 2021;

Published Online: 22 October 2021

J. B. Tuynman, MD, PhD

e-mail: j.tuynman@amsterdamumc.nl
Results. Patients treated for peritoneal and liver metastases (PMLM group) had a pooled mean survival of 26.4 months (95\% confidence interval [CI] 22.4-30.4 months), with a 3-year survival rate of $34 \%$ (95\% CI $26.7-42.0 \%$ ) and a 5-year survival rate of $25 \%$ (95\% CI $17.3-33.8 \%$ ). Surgical complications occurred more frequently for these patients than for those with peritoneal metastasis only ( $40 \%$ vs $22 \% ; p=0.0014$ ), but the mortality and reoperation rates did not differ significantly.

Conclusion. This systematic review showed that CRS and HIPEC combined with local treatment of limited liver metastasis for selected patients is feasible, although with increased morbidity and an association with a long-term survival rate of $25 \%$, which is unlikely to be achievable with systemic treatment only.

Colorectal cancer (CRC) is the third most common cancer in the world and the second cause of cancer-related death, with almost 2 million new cases worldwide every year. ${ }^{1}$ Metastases are present at diagnosis in 20 to $25 \%$ of cases, and for another 20 to $25 \%$ of patients, metastatic disease develops after initial surgical treatment. ${ }^{2,3}$ For the majority of these patients, multiple organs are involved. ${ }^{4}$ The liver is most commonly affected, followed by the peritoneum and lungs. ${ }^{5}$ When distant metastases are present, the quantity and location significantly affect prognosis, as well as the suitability for local treatment. For 
patients with liver lesions amenable to local ablative and/or surgical treatment, 5-year overall survival (OS) rates of more than $60 \%$ have been reported. ${ }^{6-10}$

Patients with peritoneal metastases (PM) from colorectal cancer have a considerably worse prognosis. Without treatment, these patients often do not survive longer than 12 months. ${ }^{11}$ Systemic therapy for peritoneal metastasis has limited effects, increasing median survival from 12 to 16 months. ${ }^{12}$ The introduction of cytoreductive surgery (CRS) and hyperthermic intraperitoneal chemotherapy (HIPEC) has resulted in favorable survival rates for selected patients who have limited disease confined to the abdominal cavity, with a median OS of 20 to 63 months ${ }^{13}$ and 5-year OS rates ranging from 23 to $52 \% .^{14}$ The survival benefit of this procedure is dependent on the severity of disease, represented by the Peritoneal Cancer Index (PCI), the completeness of cytoreduction (CC), and the histopathology of the tumor. ${ }^{13}$ These factors form the basis for the procedure's current eligibility criteria specifying that the extent of peritoneal disease must not exceed a certain PCI (commonly used in practice: PCI $<16-20$ ), that adequate cytoreduction must be possible, and that signet ring cell histopathology must be a relative contraindication.

Currently, synchronous hematogenous liver, lung, or other distant metastases generally are considered a contraindication for CRS-HIPEC. ${ }^{15}$ For patients who do not meet the selection criteria for CRS-HIPEC, disease generally is considered non-curable. Therefore, palliative systemic therapy is their sole treatment option.

Currently, centers demonstrate large heterogeneity in whether combined CRS-HIPEC and local ablation of liver metastases is offered. If it is offered, CRS-HIPEC is performed only for selected cases (e.g., for young, fit patients with a low PCI and only one or two liver lesions without signet ring cell histology). Cohort studies have shown beneficial effects of HIPEC combined with surgical/radiologic ablation of liver metastasis for these highly selected patients. A meta-analysis by Hallam et al. ${ }^{13}$ has shown that surgically treated hepatic metastasis is not predictive for overall survival after a CRS-HIPEC procedure. In 2007, Esquivel et al. ${ }^{16}$ released a consensus statement compiled in collaboration with field experts from all around the world asserting that a patient may be considered for CRSHIPEC when no more than three resectable, small hepatic lesions are present. However, due to lack of robust data, current guidelines do not offer clinical directives toward local treatment for patients with hepatic oligometastases and peritoneal metastases. Consequently, palliation with systemic therapy still is considered the only meaningful standard therapy.
This systematic review and meta-analysis of the literature aimed to determine the feasibility and clinical longterm outcome of CRS-HIPEC combined with local treatment for patients with both peritoneal and limited hepatic metastases.

\section{METHODS}

The performance and reporting of this systematic review adhere to the Preferred Reporting Items for Systematic Reviews and Meta-Analyses (PRISMA) statement (www. prisma-statement.org). ${ }^{17}$ The review protocol was registered at PROSPERO (www.crd.york.ac.uk/PROSPERO), where it can be found under registration number CRD42021219836.

\section{Search Strategy}

After scoping searches, four bibliographic databases (PubMed, Embase.com, Clarivate Analytics/Web of Science Core Collection, Wiley/Cochrane Library) were searched systematically for relevant literature from inception of the review to 9 November 2020. The searches were devised in collaboration with a medical information specialist (K.A.Z.). Search terms including synonyms, closely related words, and keywords such as "metastatic colorectal cancer" and "HIPEC" were used as index terms or freetext words. The searches contained no search filter, date, or language restrictions that would limit results to a specific study design, date, or language. Duplicate articles were excluded using Endnote (X9.3.3, AED method and Bramer method). ${ }^{18,19}$ The full search strategy used for each database is outlined in the supplementary material (Tables 1, 2, 3 , and 4). In addition to the database searches, reference lists of the included full-text articles were screened to identify additional relevant articles.

\section{Screening Process}

One reviewer (M.P.) screened all potentially relevant titles and abstracts for eligibility, selecting those that described patients treated with CRS-HIPEC who had metastases at sites other than the peritoneum. Each full-text article then was independently screened by at least two members of the review team (M.P., M.B., L.H.). Studies were included if they met the criteria, which specified (1) types of studies (randomized control studies [RCTs], cohort studies, case-control studies, and cross-sectional studies; (2) types of participants (patients with primary colorectal cancer metastasized to the peritoneum and one hematogenous site); and (3) types of interventions (studies that involved multimodal treatment with both CRS-HIPEC 


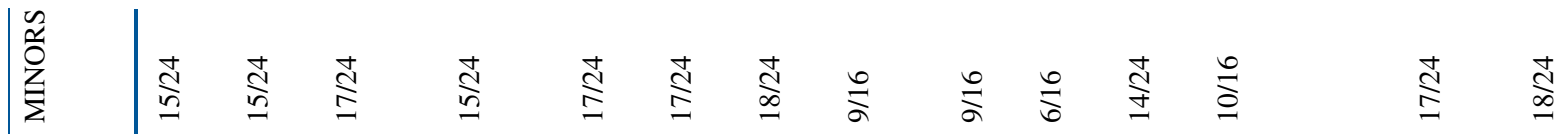

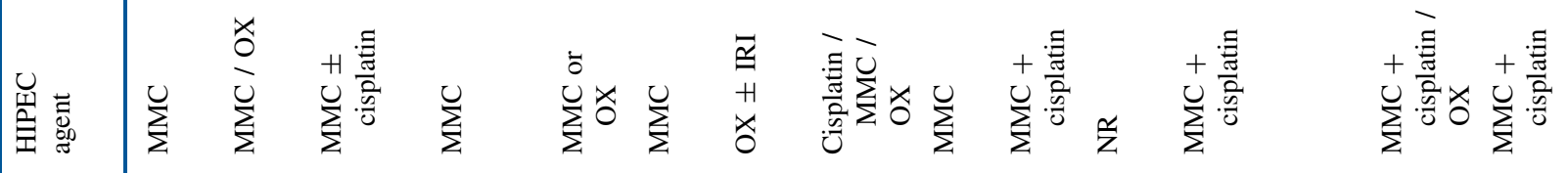 章

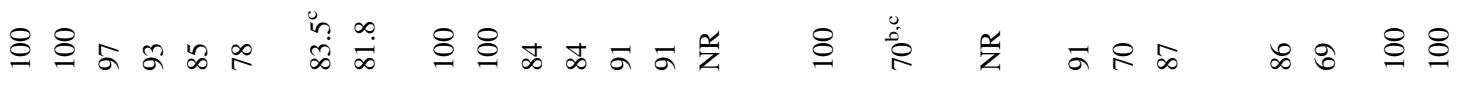

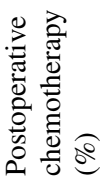

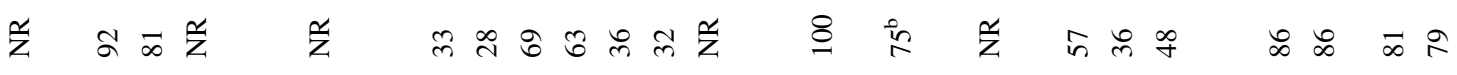

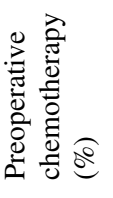 ล의

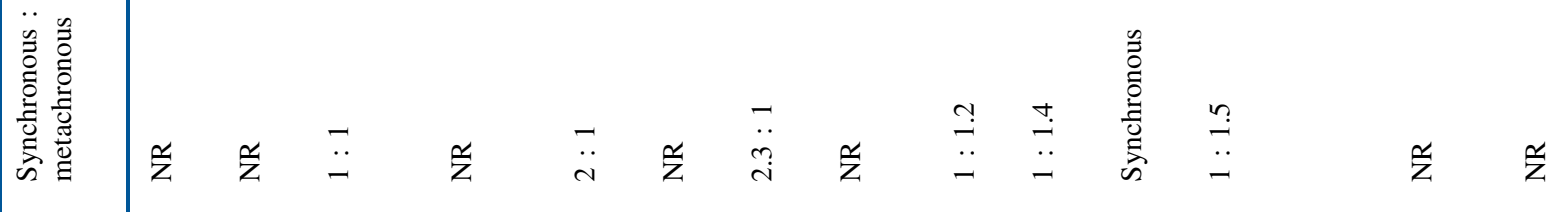

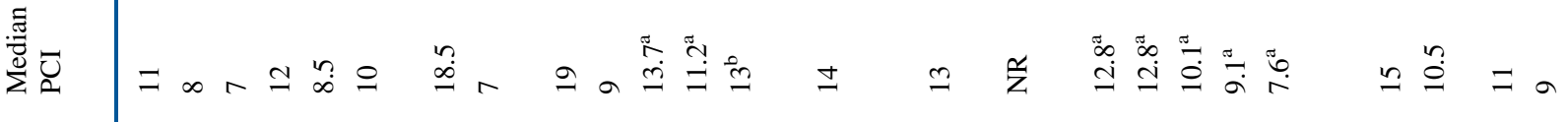

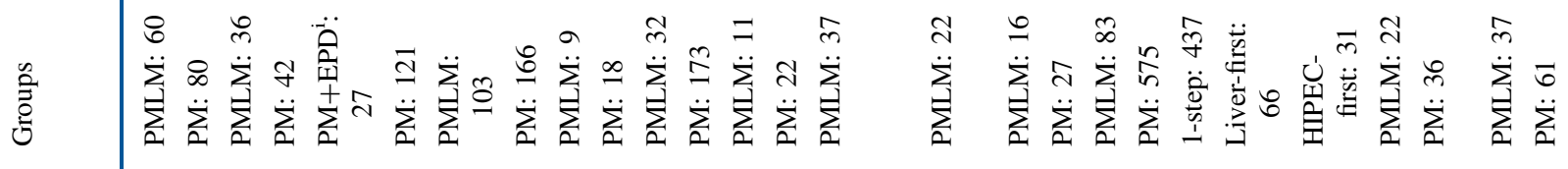

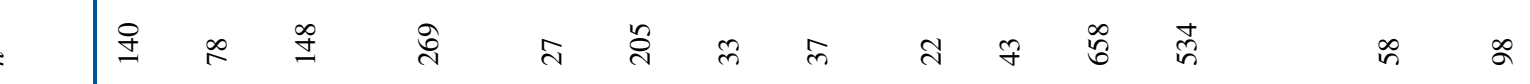




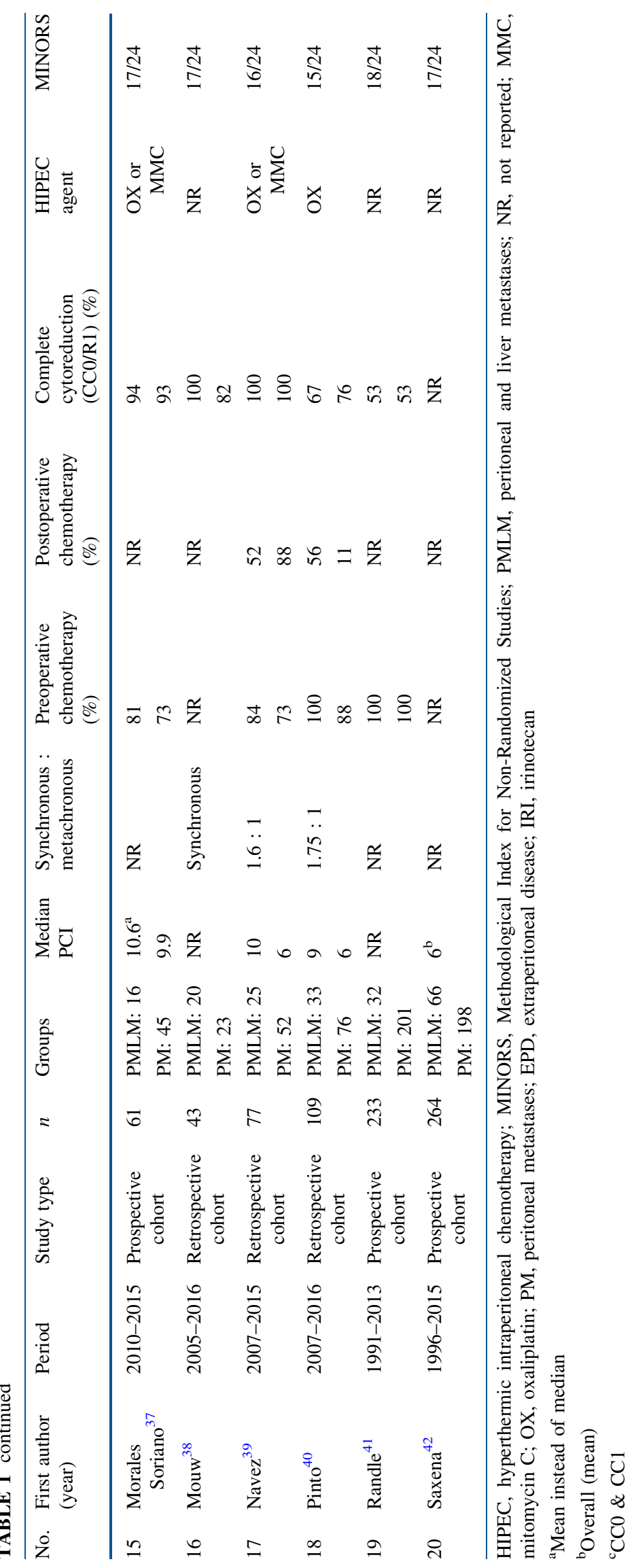


TABLE 2 Liver treatment details

TABLE 3 Long-term oncologic outcomes for the PMLM group

\begin{tabular}{|c|c|c|c|}
\hline First author (year) & No. of liver lesions & LM treatment & Preoperative chemotherapy (\%) \\
\hline Adileh $^{23}$ & NR & Resection & 97 \\
\hline Alzahrani $^{24}$ & $\begin{array}{l}<3: 25 \\
\geq 3: 11\end{array}$ & Resection & 92 \\
\hline Baratti $^{25}$ & Mean 2.38 & Resection and/or RFA & 81 \\
\hline Berger $^{26}$ & NR & Resection & 58 \\
\hline Delhorme $^{27}$ & Median 1 & Resection and/or RFA & 100 \\
\hline Downs-Canner ${ }^{28}$ & $\begin{array}{l}1: 16 \text { pts } \\
2: 7 \text { pts } \\
3 \text { or more: } 7\end{array}$ & Resection and/or RFA & 97 \\
\hline Duraj $^{29}$ & Median 1 & Resection & 90 \\
\hline Horvath $^{30}$ & $\begin{array}{l}1-2: 24 \mathrm{pts} \\
>2: 4 \mathrm{pts}\end{array}$ & Resection & 78 \\
\hline Jeon $^{31}$ & Median: 3 & Resection and/or RFA & 91 \\
\hline Kianmanesh $^{32}$ & NR & Resection & $70^{\mathrm{a}}$ \\
\hline $\mathrm{Lee}^{33}$ & NR & Resection & 59 \\
\hline Lo $\mathrm{Dico}^{34}$ & Median: 1 & Resection & 76 \\
\hline Lorimier $^{35}$ & Mean 1.9 & Resection and/or RFA & 86 \\
\hline Maggiori $^{36}$ & Median: 2 & Resection and/or RFA & NR \\
\hline Morales Soriano ${ }^{37}$ & Mean 1.2 & Resection and/or RFA & 81 \\
\hline Mouw $^{38}$ & NR & resection & NR \\
\hline Navez $^{39}$ & $\leq 3$ & Resection and/or RFA & 84 \\
\hline Pinto $^{40}$ & NR & Resection and/or RFA & 100 \\
\hline Randle $^{41}$ & NR & NR & 100 \\
\hline Saxena $^{42}$ & $\begin{array}{l}1: 34 \text { pts } \\
2-3: 30 \text { pts } \\
4 \text { or more: } 6\end{array}$ & NR & NR \\
\hline
\end{tabular}

LM, local metastasis; NR, not reported; RFA, radiofrequency ablation; pts, patients

${ }^{\mathrm{a} O v e r a l l}$

\begin{tabular}{llll}
\hline Outcome & Pooled mean $(95 \%$ CI) & No. of studies & No. of patients \\
\hline DFS (months) & $10.8(8.0-13.6)$ & 10 & 281 \\
3-Year DFS (\%) & $14.4(8.3-23.8)$ & 10 & 276 \\
Recurrence rate (\%) & & & \\
Overall & $71.8(64.7-77.9)$ & 11 & 809 \\
Peritoneum & $32.4(26.4-39.2)$ & 9 & 763 \\
Liver & $33.2(26.8-40.4)$ & 9 & 763 \\
Lung & $23.1(18.1-29.0)$ & 6 & 683 \\
Other & $15.9(9.0-26.6)$ & 8 & 406 \\
3-Year survival (\%) & $33.9(26.7-42.0)$ & 13 & 384 \\
5-Year survival (\%) & $24.6(17.3-33.8)$ & 12 & 399 \\
OS (months) & $26.4(22.4-30.4)$ & 14 &
\end{tabular}

PMLM, peritoneal and liver metastases; CI, confidence interval; DFS, disease-free survival; OS, overall survival

and a local [ablative] treatment). Any local treatment performed with the intention to remove or eradicate all tumor cells was accepted. The review excluded letters to the editor, animal studies, in vitro/ex vivo studies, case reports, (systematic) reviews, and meta-analyses. Studies reporting on fewer than 10 patients also were excluded from the 
TABLE 4 Disease-free survival at the 5-year follow-up evaluation

\begin{tabular}{ll}
\hline Study & $n /$ total $(\%)$ \\
\hline Delhorme $^{27}$ & $1 / 9(11.1)$ \\
Downs-Canner $^{28}$ & $2 / 32(6.3)$ \\
Lorimier $^{35}$ & $0 / 22(0)$ \\
Maggiori $^{36}$ & $1 / 37(2.7)$ \\
Pinto $^{40}$ & $8 / 33(24.2)$ \\
Pooled mean:\% (95\% CI) & $6.3(2.0-18.6)$ \\
\hline
\end{tabular}

CI, confidence interval

analysis. The location and study period of all the included articles were compared to identify duplicate data, and if (partially) overlapping data were found, only the study describing the largest group of patients was included.

\section{Data Extraction and Quality Assessment}

Data were collected from the included articles using a data extraction form. The completed forms were used to pool the data and to perform statistical analyses. Based on the collected data, the results were organized into two groups: a group of patients with peritoneal metastasis (PM) only and a group of patients with PM and simultaneous liver metastasis (PMLM).

The main outcomes of the analysis were overall survival (OS) and disease-free survival (DFS), calculated from the date of CRS-HIPEC. The secondary end points were perioperative outcomes, including morbidity and mortality. Major morbidity was defined as the presence of a complication classified as Clavien-Dindo grade 3 or higher.

For a proper comparison of studies, data on follow-up length, CC, PCI, and pre- and postoperative chemotherapy also were recorded. Risk of bias in individual studies was assessed using the Methodological Index for Non-Randomized Studies (MINORS). ${ }^{20}$

\section{Statistical Analysis}

Meta-analysis was performed for both perioperative and survival outcomes in the PMLM group. To determine the feasibility of the combined treatment, a meta-analysis of perioperative outcomes for the PM group was performed using formal statistical comparison with the PMLM group. Only studies that provided appropriate data were included in the meta-analysis for perioperative outcomes.

Major morbidity and mortality risk ratios (RRs) were calculated using the number of events and the numbers of patients at risk, which also was how proportional survival outcomes such as 3- and 5-year overall survival data were pooled. For the survival outcomes, measured in months, means, and standard deviations, were calculated using the method described by Wan et al., ${ }^{21}$ after which these means were pooled. This was performed only for the PMLM group due to the objective of this study. A random-effects model was applied in all analyses due to large heterogeneity. All analyses were performed using the meta package in $\mathrm{R}^{22}$

\section{RESULTS}

\section{Study Selection}

The literature search generated 2621 references: 463 in PubMed, 1403 in Embase.com, 698 in Clarivate Analytics/ Web of Science Core Collection, and 57 in Wiley/ Cochrane library. After removal of reference duplicates selected from more than one database, 1218 references remained, all of which were included in the title/abstract screening. Of these articles, 54 were included for full-text assessment, together with an additional 10 articles identified from their reference lists. This resulted in 20 articles included for the qualitative synthesis, 16 of which presented sufficient data to be included in the quantitative synthesis. The search and selection process is illustrated in Fig. 1.

\section{Study Characteristics}

In total, 3137 patients participated in 2 case-control studies, 11 prospective studies, and 7 retrospective cohort studies. ${ }^{23-42}$ No randomized studies were identified. Although the search and selection process was designed to include various oligometastatic sites, the studies that met the criteria for inclusion all presented liver metastases. With the exception of the studies by Baratti et al. ${ }^{25}$ and Lo Dico et al., ${ }^{34}$ the studies in this review exclusively presented patients treated in a one-step procedure, with CRSHIPEC and liver resection/ablation performed during one surgical procedure.

Relatively high variability in PCI, CC, and perioperative systemic therapy protocols was found among the studies (Table 1). In most of the studies, the PMLM patients had a higher PCI than the PM-only patients. In the vast majority of the studies, the PCI was below 20, which corresponds with clinical guidelines. The $\mathrm{CC}$ rates varied between 53 and $100 \%$, with a mean of $88 \%$ in the PMLM group and $80 \%$ in the PM-only group. The majority of the participants received pre- and/or postoperative chemotherapy, with a higher proportion in the preoperative setting for the PMLM group ( $(78 \%$ vs $62 \%)$ and similar percentages in the PMonly group (60\% vs $61 \%)$. The most frequently used 
FIG. 1 Search and selection process (PRISMA flowchart)

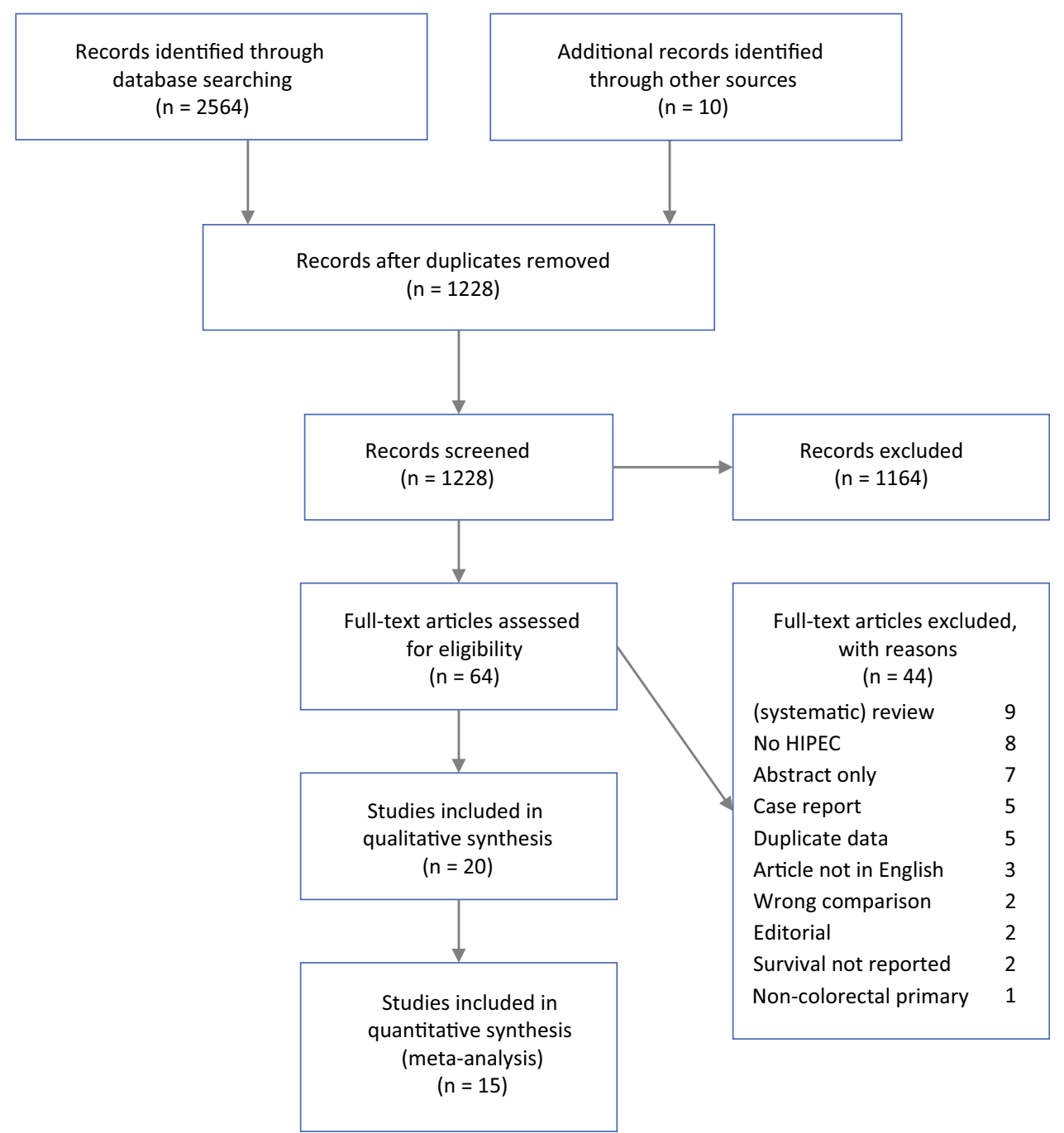

chemotherapeutic agent for HIPEC was mitomycin C, either with or without cisplatin, followed by oxaliplatin.

\section{Survival Outcomes}

Only a small proportion of the trials $(n=13)$ reported the extent of liver disease. Liver disease was limited in the majority of patients, as described by either a low number of lesions or a limited resection. The methods used to treat the hematogenous metastases included resection and radiofrequency ablation (RFA). Details of the liver treatment are presented in Table 2.

Table 3 shows data on the long-term oncologic outcomes in the PMLM group. Overall, $72 \%$ of the patients experienced recurrence. These recurrences most frequently occurred in the peritoneum (32\%) or liver (33\%), followed by pulmonary dissemination (23\%), and $16 \%$ of the recurrences developed at another site. The pooled mean time to detection of these recurrences (DFS) was 10.8 months (95\% confidence interval [CI] 8.0-13.6 months).
After 3 years, a pooled proportion of $14 \%$ of the patients (95\% CI 8.3-23.8\%) were disease-free (13 studies involving 406 patients). A small percentage of the patients (6\%) still showed no evidence of disease after 5 years, as shown in Table 4 . The pooled mean overall survival was 26.4 months (95\% CI 22.4-30.4 months), and the pooled 3and 5-year overall survival rates were $34 \%$ and $25 \%$, respectively. Finally, Table 5 shows an overview of the PCI, CC score, and median OS for all the studies.

\section{Perioperative Outcomes}

As shown in Table 6, major morbidity due to surgery was reported in 11 studies, with rates ranging from 12 to $75 \%$ in the PMLM group versus $9 \%$ to $40 \%$ in the PM-only group, and averages of $40 \%$ and $22 \%$, respectively (RR, $1.78 ; p=0.0014)$. Pooled mortality tended to be higher in the PMLM group (5\% vs $2 \% ; p=0.0956$ ), but the difference was not significant, similar to the reoperation rate ( $21 \%$ vs $16 \% ; p=0.2744)$. The mean hospital and 
TABLE 5 Overview of PCI, CC score, and OS for both groups

\begin{tabular}{|c|c|c|c|c|}
\hline Study & Group & Median PCI & $\begin{array}{l}\text { Complete cytoreduction }(\mathrm{CC} 0 / \mathrm{R} 1) \\
(\%)\end{array}$ & Median OS \\
\hline \multirow[t]{2}{*}{ Alzahrani $^{24}$} & PMLM: 36 & 7 & 97 & 24.4 \\
\hline & PM: 42 & 12 & 93 & 45.5 \\
\hline \multirow[t]{2}{*}{ Baratti $^{25}$} & PM+EPD: 27 & 8.5 & 85 & 19.0 \\
\hline & PM: 121 & 10 & 78 & 60.1 \\
\hline \multirow[t]{2}{*}{ Berger $^{26}$} & PMLM: 103 & 18.5 & $83.5^{\mathrm{a}}$ & $45.1^{\mathrm{b}}$ \\
\hline & PM: 166 & 7 & 81.8 & $73.5^{\mathrm{b}}$ \\
\hline \multirow[t]{2}{*}{ Delhorme $^{27}$} & PMLM: 9 & 19 & 100 & 27.6 \\
\hline & PM: 18 & 9 & 100 & 39.1 \\
\hline \multirow[t]{2}{*}{ Downs-Canner ${ }^{28}$} & PMLM: 32 & $13.7^{\mathrm{c}}$ & 84 & 13 \\
\hline & PM: 173 & $11.2^{\mathrm{c}}$ & 84 & 20.5 \\
\hline \multirow[t]{2}{*}{ Duraj $^{20}$} & PMLM: 11 & $13^{\mathrm{d}}$ & 91 & 15 \\
\hline & PM: 22 & & 91 & 34 \\
\hline Horvath $^{30}$ & PMLM: 37 & 14 & NR & 22 \\
\hline Jeon (2019) & PMLM: 22 & 13 & 100 & 16.7 \\
\hline \multirow[t]{2}{*}{ Kianmanesh $^{32}$} & PMLM: 16 & NR & $70^{\mathrm{a}, \mathrm{d}}$ & 36.0 \\
\hline & PM: 27 & & & 35.3 \\
\hline \multirow[t]{3}{*}{ Lo $\mathrm{Dico}^{34}$} & 1-step: 437 & $10.1^{\mathrm{c}}$ & 91 & $44.8^{\mathrm{b}}$ \\
\hline & Liver-first: 66 & $9.1^{\mathrm{c}}$ & 70 & $63.7^{\mathrm{b}}$ \\
\hline & HIPEC-first: 31 & $7.6^{\mathrm{c}}$ & 87 & $52.6^{\mathrm{b}}$ \\
\hline \multirow[t]{2}{*}{ Lorimier $^{35}$} & PMLM: 22 & 15 & 86 & $36.1^{\mathrm{b}}$ \\
\hline & PM: 36 & 10.5 & 69 & $25.2^{\mathrm{b}}$ \\
\hline \multirow[t]{2}{*}{ Maggiori $^{36}$} & PMLM: 37 & 11 & 100 & 32 \\
\hline & PM: 61 & 9 & 100 & 49 \\
\hline \multirow[t]{2}{*}{ Morales Soriano $^{37}$} & PMLM: 16 & $10.6^{\mathrm{c}}$ & 94 & 36 \\
\hline & PM: 45 & 9.9 & 93 & 33 \\
\hline \multirow[t]{2}{*}{ Navez $^{39}$} & PMLM: 25 & 10 & 100 & 27.5 \\
\hline & PM: 52 & 6 & 100 & 59.2 \\
\hline \multirow[t]{2}{*}{ Pinto $^{40}$} & PMLM: 33 & NR & 67 & 31 \\
\hline & PM: 76 & & 76 & 65 \\
\hline \multirow[t]{2}{*}{ Randle $^{41}$} & PMLM: 32 & NR & 53 & 21.2 \\
\hline & PM: 201 & & 53 & 33.6 \\
\hline \multirow[t]{2}{*}{ Saxena $^{42}$} & PMLM: 66 & $6^{\mathrm{d}}$ & NR & 32.3 \\
\hline & PM: 198 & & & 30.5 \\
\hline
\end{tabular}

PCI, peritoneal cancer index; CC, completeness of cytoreduction; OS, overall survival; PMLM, peritoneal and liver metastases; PM, peritoneal metastases; EPD, extraperitoneal disease NR, not reported; HIPEC, hyperthermic intraperitoneal chemotherapy

${ }^{\mathrm{a}} \mathrm{CCO} \& \mathrm{CC} 1$

${ }^{\mathrm{b}}$ Calculated from date of diagnosis

${ }^{c}$ Mean instead of median

dOverall (mean)

\begin{tabular}{llllllll}
\hline Outcome & PMLM & & & PM & & RR & $p$ Value \\
\cline { 2 - 3 } & $\%(95 \% \mathrm{CI})$ & $n$ & & $\%(95 \% \mathrm{CI})$ & & & \\
\hline Major morbidity & $39.9(29.2-51.7)$ & 291 & & $22.0(16.7-28.4)$ & 688 & 1.78 & 0.0014 \\
Mortality & $5.3(2.9-9.6)$ & 198 & & $2.0(0.8-4.7)$ & 532 & 2.7 & 0.0956 \\
Reoperation & $21.2(16.0-27.6)$ & 189 & & $16.0(9.0-27.0)$ & 921 & 1.38 & 0.2744 \\
\hline
\end{tabular}

PMLM, peritoneal and liver metastases; PM, peritoneal metastases; RR, risk ratio

${ }^{\mathrm{a}}$ Data are displayed as pooled mean $(95 \%$ confidence interval)
TABLE 6 Perioperative outcomes $^{\mathrm{a}}$ 
intensive care unit (ICU stays could not be reliably calculated due to the confounding effect of outliers in the individual study data. The median hospital stay ranged from 13 to 28 days in the PMLM group and from 9 to 25 days in the PM group. The ICU stay was 1 to 12 days in the PMLM group and 1 to 16 days in the PM group.

\section{DISCUSSION AND CONCLUSION}

This systematic review and meta-analysis show that combined local treatment of peritoneal and hepatic metastases for selected patients is feasible, resulting in a pooled mean overall survival period of 26.4 months and a pooled 5-year overall survival rate of $25 \%$. These survival outcomes suggest an improvement compared with literature reporting on similar patients who received only systemic therapy, ${ }^{11,12,43,44}$ although a formal comparison could not be made because the available literature lacked studies comparing combined local treatment and systemic therapy. These data provide a lead to justify combined CRS-HIPEC and local ablative treatment for patients with both limited liver and peritoneal metastasis as an alternative for systemic treatment only and form a basis for prospective studies that further elucidate the role of local treatment of combined metastatic sites.

The specific group of patients who have both peritoneal and limited hepatic lesions treated with current standard systemic therapy is not well described in literature, and no comparative studies with surgical treatment are available at all. Several non-comparative studies describe patients treated with systemic therapy for peritoneal dissemination and any amount of systemic disease, without specification of location or extent. Pooled individual data of these patients from the Analysis and Research in Cancers of the Digestive System (ARCAD) database, which contains data from multiple randomized chemotherapy trials, show a median survival period of 12.6 months (95\% CI 12.0-13.1 months). ${ }^{12}$

Another large population-based study by Simkens et al. ${ }^{11}$ that included almost 2500 patients with peritoneal and systemic metastases reported a median survival range of 6.8 to 13.3 months for patients treated with systemic therapy, depending on tumor histology. Furthermore, Pelz et al. $^{43}$ and Razenberg et al. ${ }^{44}$ presented results with a median survival of 8.0 to 9.9 months for a mixed treatment population.

It should be noted that the average disease burden (regarding location and volume) in these groups likely was higher than in the studies included in this review of patients who received surgical treatments. In addition, younger patients in good physical condition tend to be less likely to receive systemic therapy only. These two factors resulted in an underestimation of survival in the systemic treatment group. Nevertheless, data from the current review showed a survival period of 26.4 months for the patients treated with CRS-HIPEC and local treatment (liver resection/radiofrequency ablation [RFA] or other ablative techniques), which at least warrants further investigation and indicates that a direct comparison is needed to determine whether an actual survival benefit exists. In addition, several studies included in this review showed long-term DFS for a small number of patients, which is unlikely to occur with systemic therapy alone.

The survival benefit is countered by the treatment-associated morbidity. Systemic therapy usually is a long-term process, during which complications might develop and worsen over time, whereas surgical complications typically are more short-term but more intense and life-threatening. As such, it is difficult to compare the burden of systemic treatment with that of surgery because the inherent risks differ in nature. Instead, a fairer comparison for determining the hazards of combined treatment is between CRSHIPEC only and CRS-HIPEC with additional liver treatment (RFA and/or resection).

This study showed that the addition of liver resection and ablation is associated with increased morbidity (RR, 1.78; $p=0.0014)$. The differences in reoperation and mortality rates, however, were not found to be significant. For patients with metastasis limited to the peritoneum, the morbidity rate for CRS-HIPEC is high but accepted by clinicians and patients because of the long-term survival benefits. Based on the results of the current study, the same could be argued for the combined treatment. Furthermore, although more complications occurred, the range of median hospital stays in the PMLM group differed only a couple of days from that in the PM-only group.

This systematic review provides an extensive overview of the current literature. However, the current literature is hampered by the quality of the data. The studies included in this review used different criteria for inclusion and exclusion of patients, and potential selection bias could not be excluded. Although not always formally asserted, physical fitness of the patient was an important factor in determining eligibility for combined treatment.

Other inclusion criteria were quite heterogeneous, which was a limitation of the current review. An example of such a criterion was the extent of liver disease. As shown in Table 2, the hepatic disease burden was low on the average, but no clear cutoff value was universally applied. This should also be a point of focus in future investigations. Especially noteworthy was the variation in the use of preoperative chemotherapy. Eight of the included studies $^{24,28,30,34-37,40}$ included only patients who did not show disease progression during or after preoperative systemic therapy. In other studies, this was not required, resulting in 
a mixed population of responders, non-responders, and patients who did not receive any preoperative chemotherapy.

In addition, the use of postoperative chemotherapy varied considerably between studies. These parameters likely influenced survival, thus resulting in heterogeneous outcomes among the various cohorts. The same could be argued about $\mathrm{CC}$, which has been identified as a prognostic factor for survival. ${ }^{13}$

Some retrospective studies limited their participants to patients with complete cytoreduction (CC-0), but this was not possible for the prospective studies. A low percentage of CC-0 within a cohort could negatively influence survival data and affect the reported outcome.

The most important weakness of the included cohort studies was the improper group comparison. Preferably, palliative systemic treatment would be compared with extensive ablative treatment for these patients, but unfortunately, such studies have not been published to date, and thus, only an indirect comparison based on the literature was possible.

In conclusion, the current study showed that it is feasible to perform CRS-HIPEC and liver resection and/or ablation for patients with both peritoneal and limited hepatic metastasis because it seems to provide favorable long-term clinical outcomes despite current international guidelines suggesting that CRS-HIPEC not be performed for these patients. However, this combined treatment needs to be more closely investigated in future trials. Participants should be selected carefully based on their physical condition and disease burden, for which specific criteria have yet to be determined. This should be considered carefully in the design of studies confirming these data for safe implementation of the combined CRS-HIPEC and liver metastasis treatment for selected patients in clinical practice.

DISCLOSURES The authors declare that they have no conflict of interest.

Supplementary Information The online version contains supplementary material available at https://doi.org/10.1245/s10434021-10925-y.

OPEN ACCESS This article is licensed under a Creative Commons Attribution 4.0 International License, which permits use, sharing, adaptation, distribution and reproduction in any medium or format, as long as you give appropriate credit to the original author(s) and the source, provide a link to the Creative Commons licence, and indicate if changes were made. The images or other third party material in this article are included in the article's Creative Commons licence, unless indicated otherwise in a credit line to the material. If material is not included in the article's Creative Commons licence and your intended use is not permitted by statutory regulation or exceeds the permitted use, you will need to obtain permission directly from the copyright holder. To view a copy of this licence, visit http://creativecommons. org/licenses/by/4.0/.

\section{REFERENCES}

1. Bray F, Ferlay J, Soerjomataram I, Siegel RL, Torre LA, Jemal A. Global cancer statistics 2018: GLOBOCAN estimates of incidence and mortality worldwide for 36 cancers in 185 countries. CA Cancer J Clin. 2018;68:394-424.

2. SEER Cancer Stat Facts: Colorectal Cancer. National Cancer Institute. Retrieved 2 April 2021 at https://seer.cancer.gov/statfac ts/html/colorect.html.

3. Kuchel A, Ahern E, Collins S, et al. Trends in epidemiology, treatment and molecular testing of metastatic colorectal cancer in a real-world multi-institution cohort study. Asia Pac J Clin Oncol. 2021;17:84-93.

4. Riihimaki M, Hemminki A, Sundquist J, Hemminki K. Patterns of metastasis in colon and rectal cancer. Sci Rep. 2016;6:29765.

5. van der Geest LG, Lam-Boer J, Koopman M, Verhoef C, Elferink MA, de Wilt JH. Nationwide trends in incidence, treatment, and survival of colorectal cancer patients with synchronous metastases. Clin Exp Metastasis. 2015;32:457-65.

6. Chakedis J, Schmidt CR. Surgical treatment of metastatic colorectal cancer. Surg Oncol Clin North Am. 2018;27:377-99.

7. Guerrera F, Mossetti C, Ceccarelli M, et al. Surgery of colorectal cancer lung metastases: analysis of survival, recurrence and resurgery. J Thorac Dis. 2016;8:1764-71.

8. Hayashi M, Inoue Y, Komeda K, et al. Clinicopathological analysis of recurrence patterns and prognostic factors for survival after hepatectomy for colorectal liver metastasis. BMC Surg. 2010;10:1-12.

9. Kim YJ, Park IJ, Kim HR, et al. Post-pulmonary metastasectomy prognosis after curative resection for colorectal cancer. Oncotarget. 2017;8:36566-77.

10. Shin H, Kim CW, Lee JL, et al. Solitary colorectal liver metastasis after curative intent surgery: prognostic factors affecting outcomes and survival. ANZ J Surg. 2019;89:61-7.

11. Simkens GA, Razenberg LG, Lemmens VE, Rutten HJ, Creemers G-J, de Hingh IH. Histological subtype and systemic metastases strongly influence treatment and survival in patients with synchronous colorectal peritoneal metastases. Eur J Surg Oncol. 2016;42:794-800.

12. Franko J, Shi Q, Meyers JP, et al. Prognosis of patients with peritoneal metastatic colorectal cancer given systemic therapy: an analysis of individual patient data from prospective randomised trials from the Analysis and Research in Cancers of the Digestive System (ARCAD) database. Lancet Oncol. 2016;17:1709-19.

13. Hallam S, Tyler R, Price M, Beggs A, Youssef H. Meta-analysis of prognostic factors for patients with colorectal peritoneal metastasis undergoing cytoreductive surgery and heated intraperitoneal chemotherapy. BJS Open. 2019;3:585-94.

14. Flood M, Narasimhan V, Waters P, et al. Survival after cytoreductive surgery and hyperthermic intraperitoneal chemotherapy for colorectal peritoneal metastases: a systematic review and discussion of latest controversies. Surgeon. 2021;18:310-20.

15. Landelijke Werkgroep Gastro Intestinale Tumoren. Colorectaalcarcinoom: Landelijke richtlijn. 2019.

16. Esquivel J, Sticca R, Sugarbaker P, et al. Cytoreductive surgery and hyperthermic intraperitoneal chemotherapy in the management of peritoneal surface malignancies of colonic origin: a consensus statement. Soc Surg Oncol Ann Surg Oncol. 2007; 14:128-33.

17. Moher D, Liberati A, Tetzlaff J, Altman DG, Group P. Preferred reporting items for systematic reviews and meta-analyses: the PRISMA statement. PLoS Med. 2009;6:e1000097. 
18. Otten R, de Vries R, Schoonmade L. Amsterdam Efficient Deduplication (AED) method. 2019.

19. Bramer WM, Giustini D, de Jonge GB, Holland L, Bekhuis T. De-duplication of database search results for systematic reviews in EndNote. J Med Libr Assoc. 2016;104:240-3.

20. Slim K, Nini E, Forestier D, Kwiatkowski F, Panis Y, Chipponi J. Methodological index for non-randomized studies (MINORS): development and validation of a new instrument. ANZ J Surg. 2003;73:712-6.

21. Wan X, Wang W, Liu J, Tong T. Estimating the sample mean and standard deviation from the sample size, median, range and/or interquartile range. BMC Med Res Method. 2014;14:135.

22. $R:$ A Language and Environment for Statistical Computing (computer program). R Foundation for Statistical Computing, Vienna, Austria, 2021.

23. Adileh M, Mor E, Assaf D, et al. Perioperative and oncological outcomes of combined hepatectomy with complete cytoreduction and hyperthermic intraperitoneal chemotherapy for metastatic colorectal cancer. Ann Surg Oncol. 2021;28:3320-29.

24. Alzahrani N, Ung L, Valle SJ, Liauw W, Morris DL. Synchronous liver resection with cytoreductive surgery for the treatment of liver and peritoneal metastases from colon cancer: results from an Australian centre. ANZ J Surg. 2017;87:E167-72.

25. Baratti D, Kusamura S, Iusco D, et al. Should a history of extraperitoneal disease be a contraindication to cytoreductive surgery and hyperthermic intraperitoneal chemotherapy for colorectal cancer peritoneal metastases? Dis Colon Rectum. 2018;61:1026-34.

26. Berger Y, Aycart S, Tabrizian P, et al. Cytoreductive surgery and hyperthermic intraperitoneal chemotherapy in patients with liver involvement. J Surg Oncol. 2016;113:432-7.

27. Delhorme JB, Dupont-Kazma L, Addeo P, et al. Peritoneal carcinomatosis with synchronous liver metastases from colorectal cancer: who will benefit from complete cytoreductive surgery? Int J Surg. 2016;25:98-105.

28. Downs-Canner S, Shuai Y, Ramalingam L, et al. Safety and efficacy of combined resection of colorectal peritoneal and liver metastases. J Surg Res. 2017;219:194-201.

29. Duraj FF, Cashin PH. Cytoreductive surgery and intraperitoneal chemotherapy for colorectal peritoneal and hepatic metastases: a case-control study. J Gastrointest Oncol. 2013;4:388-96.

30. Horvath P, Beckert S, Königsrainer A, Nadalin S, Königsrainer I. Cytoreductive surgery and hyperthermic intraperitoneal chemotherapy combined with liver resection for concurrent peritoneal and hepatic metastases of gastrointestinal and gynecological primary tumors. J Visc Surg. 2019;156:475-84.

31. Jeon Y, Park EJ, Lim JH, Baik SH. Clinical outcomes of complete cytoreduction with concurrent liver resection followed by hyperthermic intraperitoneal chemotherapy for synchronous peritoneal and liver metastatic colorectal cancer. World J Surg Oncol. 2019;17:214.

32. Kianmanesh R, Scaringi S, Sabate JM, et al. Iterative cytoreductive surgery associated with hyperthermic intraperitoneal chemotherapy for treatment of peritoneal carcinomatosis of colorectal origin with or without liver metastases. Ann Surg. 2007;245:597-603.

33. Lee RM, Gamboa AC, Turgeon MK, et al. A novel preoperative risk score to optimize patient selection for performing concomitant liver resection with cytoreductive surgery/HIPEC. $J$ Surg Oncol. 2021;123:187-95.

34. Lo Dico R, Faron M, Yonemura Y, et al. Combined liver resection and cytoreductive surgery with HIPEC for metastatic colorectal cancer: results of a worldwide analysis of 565 patients from the Peritoneal Surface Oncology Group International (PSOGI). Eur J Surg Oncol. 2021;47:89-100.

35. Lorimier G, Linot B, Paillocher N, et al. Curative cytoreductive surgery followed by hyperthermic intraperitoneal chemotherapy in patients with peritoneal carcinomatosis and synchronous resectable liver metastases arising from colorectal cancer. Eur $J$ Surg Oncol. 2017;43:150-8.

36. Maggiori L, Goere D, Viana B, et al. Should patients with peritoneal carcinomatosis of colorectal origin with synchronous liver metastases be treated with a curative intent? A case-control study. Ann Surg. 2013;258:116-21.

37. Morales Soriano R, Morón Canis JM, Molina Romero X, et al. Influence of simultaneous liver and peritoneal resection on postoperative morbi-mortality and survival in patients with colon cancer treated with surgical cytoreduction and intraperitoneal hyperthermic chemotherapy. Cir Esp. 2017;95:214-21.

38. Mouw TJ, Lu J, Woody-Fowler M, et al. Morbidity and mortality of synchronous hepatectomy with cytoreductive surgery/hyperthermic intraperitoneal chemotherapy (CRS/HIPEC). $J$ Gastrointest Oncol. 2018;9:828-32.

39. Navez J, Remue C, Leonard D, et al. Surgical treatment of colorectal cancer with peritoneal and liver metastases using combined liver and cytoreductive surgery and hyperthermic intraperitoneal chemotherapy: report from a single-centre experience. Ann Surg Oncol. 2016;23:666-73.

40. Pinto A, Hobeika C, Philis A, Kirzin S, Carrère N, Ghouti L. Synchronous liver metastases and peritoneal carcinomatosis from colorectal cancer: different strategies for curative treatment? Langenbecks Arch Surg. 2019;404:477-88.

41. Randle RW, Doud AN, Levine EA, et al. Peritoneal surface disease with synchronous hepatic involvement treated with cytoreductive surgery (CRS) and hyperthermic intraperitoneal chemotherapy (HIPEC). Ann Surg Oncol. 2015;22:1634-8.

42. Saxena A, Valle SJ, Liauw W, Morris DL. Limited synchronous hepatic resection does not compromise perioperative outcomes or survival after cytoreductive surgery and hyperthermic intraperitoneal chemotherapy. J Surg Oncol. 2017;115:417-24.

43. Pelz JOW, Chua TC, Esquivel J, et al. Evaluation of best supportive care and systemic chemotherapy as treatment stratified according to the retrospective Peritoneal Surface Disease Severity Score (PSDSS) for peritoneal carcinomatosis of colorectal origin. BMC Cancer. 2010;10.

44. Razenberg LGEM, Lemmens VEPP, Verwaal VJ, et al. Challenging the dogma of colorectal peritoneal metastases as an untreatable condition: results of a population-based study. Eur J Cancer. 2016;65:113-20.

Publisher's Note Springer Nature remains neutral with regard to jurisdictional claims in published maps and institutional affiliations. 